\title{
Estimating The Gravitational Potential from IR Images
}

\author{
A. C. Quillen \\ Astronomy Department, Ohio State University, 174 W. 18th Ave, \\ Columbus, $\mathrm{OH} 43210$
}

\begin{abstract}
Detailed studies of stellar and gas dynamics have been carried out primarily in model galaxies which are not good approximations to the shapes of real galaxies (Athanassoula 1991). The high quality of recently available near-IR images of galaxies makes it possible to make realistic mass models of the matter that is traced by starlight. The gravitational potential derived from the mass model of a galaxy can be used to conduct stellar and gas dynamical studies in the galaxy, and the results can be compared to observed velocity fields and other observations of these galaxies. In this report we discuss how accurately the gravitational potential can be estimated based on an infrared image of the galaxy. We describe two uses of such an estimate of a gravitational potential: stellar orbits integrated in the barred galaxy NGC 4314 and an estimate of the gas inflow rate along the bar in NGC 7479.
\end{abstract}

\section{Observations}

Galaxies look different in the near-IR $(J, H, K)$ than in the optical $(B, V, R)$ bands (see Figures 1 and 2). Note that the near-IR images are smoother or have less small scale structure than the optical images. This is real since the images shown are presented at the same spatial resolution. The optical and infrared wavelengths have different sensitivity to extinction from dust and are dominated by light from different stellar populations.

Extinction from dust is significantly lower in the near-IR $\left(A_{K} \sim 0.1 A_{V}\right)$ than at optical wavelengths. Dust typically has a lower vertical scale height than the stars in a galaxy so that when dust is concentrated in sharp features in the plane of the galaxy such as along spiral arms or along a bar, a sharp absorption feature can be seen.

The lower infrared extinction also makes it possible to detect underlying features such as stellar rings, spiral arms, bars, and nuclei that may be difficult to see in optical images - this is particularly true in the central regions of galaxies where there may be a high column depth of dust (for example see Figure 2 showing the nuclear spiral and bar in NGC 1097 which is apparent in the nearIR).

Near-IR images of galaxies detect light primarily from cool giants and dwarfs that contribute a major fraction of the bolometric luminosity of a galaxy. Particularly in spiral galaxies, these stars are much better tracers of the mass distribution of the galaxy than are the bluer, hotter stars (Frogel 1988). This older 


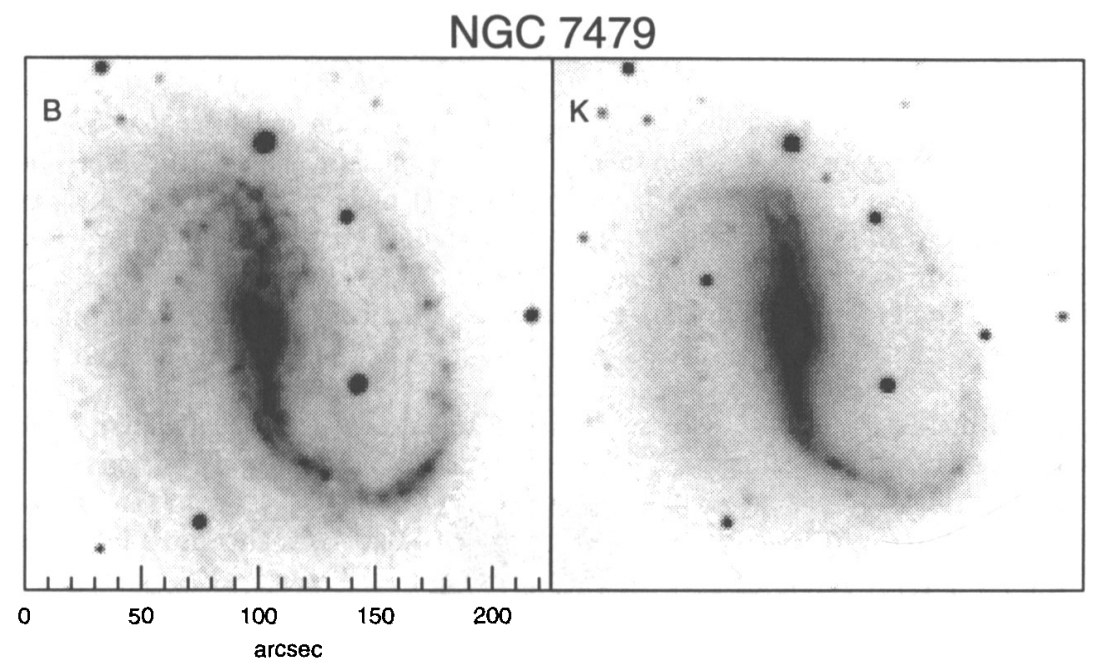

Figure $1 . \quad B$ and $K$ band images of NGC 7479 .

population is typically more evenly distributed than young blue stars which can be concentrated in linear features following spiral arms, rings or bars. This older population also should have a higher vertical scale height and larger velocity dispersion than newly formed stars, so that the galaxy appears smoother when viewed in the near-IR.

We can make the assumption that near-IR images are a good tracer of mass (note that in cases of high recent starbursts, supergiants can contribute at $2.2 \mu \mathrm{m}$ ( $K$ band) so in these situations $K$ band light may not be an ideal tracer of mass). Recent comparison of IR generated to observed $\mathrm{H} \alpha$ rotation curves (Heraudeau et al. 1995) confirms that in the central regions of late-type galaxies (central few exponential scale lengths) IR maximal disk models are usually successful at reproducing rotation curves.

\section{Estimating the Gravitational Potential from Images}

The major force on gas and stars in galaxies is the gravitational potential. An estimate of the gravitational potential in a galaxy can be used for a variety of studies, for example:

- Orbits in barred galaxies

Kent \& Glaudell (1989): stellar orbits in the barred galaxy NGC 936

Quillen et al. (1994): stellar orbits in the barred galaxy NGC 4314

Patsis et al. (1995): a study of orbital families in NGC 4314

- Estimating the torque on the gas and the gas inflow rate

Quillen et al. (1995a) in NGC 7479 


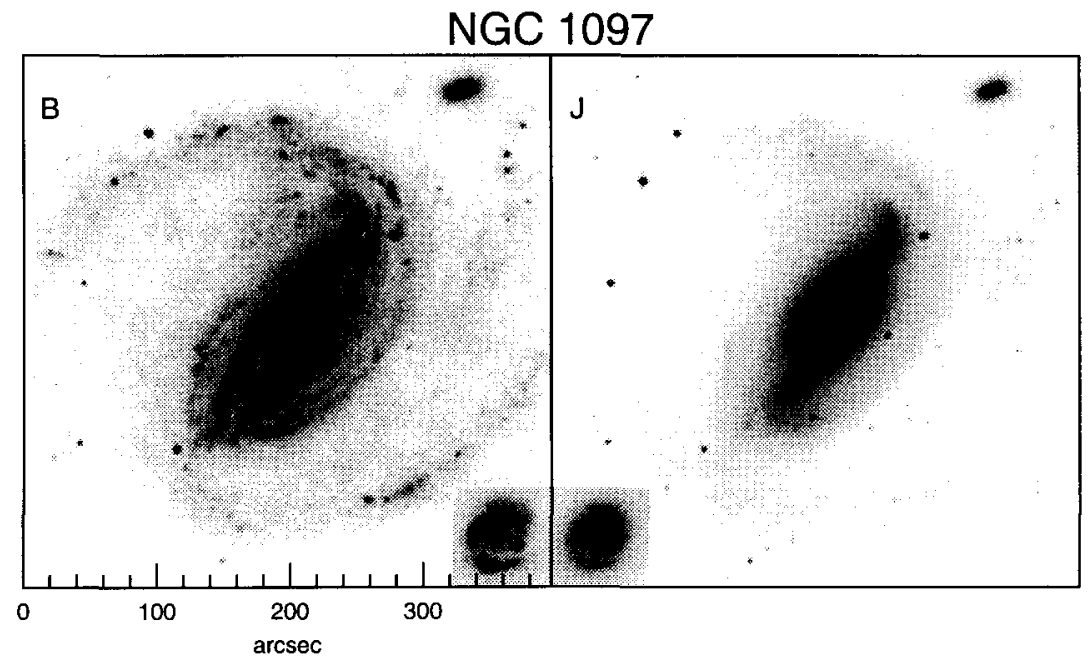

Figure 2. $\quad B$ and $J$ band images of NGC 1097. Images in the lower central part of the figure are $B$ and $J$ images of the nuclear ring and are at $1 / 2$ scale of the larger images.

- Estimating the torque on the stars and the stellar heating rate Gnedin, Goodman \& Rhoads (1995) in M100

- Using the potential as a basis for fluid simulations

Florida, Paris and Maryland groups all working on the simulation of the fluid dynamics of the ISM in NGC 7479 with SPH, sticky particle and hydro fluid codes respectively.

Lindblad, Lindblad \& Athanassoula (these proceedings) hydro simulation of the HI in NGC 1365

The gravitational potential is given by the convolution of the mass (or light) density with the function $1 / r$. Our images are observed on a grid so that a quick way to estimate the potential is by using an FFT to do the convolution and no assumption about the shape of the galaxy is required. However, our galaxy images are two-dimensional not three-dimensional, so some assumption about the vertical structure of the galaxy is needed. Observations of edge-on galaxies find that scale heights of disks are constant as a function of radius (see references cited in Quillen et al. 1994; in the future it would be useful if the vertical structures of $3 \mathrm{D} \mathrm{N}$-body simulations are described in detail, so that we can determine how bad an assumption this is). If we assume that the scale height is constant across the disk of the galaxy we can estimate the potential by convolving our images with a function $g(r)$ that is

$$
g(r)=\int_{-\infty}^{\infty} \frac{\rho_{z}(z) d z}{\sqrt{r^{2}+z^{2}}}
$$


where $\rho_{z}(z)$ describes the vertical density distribution of the disk. The function $g(r)$ is equivalent to $1 / r$ for $r \gg h$ where $h$ is the vertical scale height of the disk, and levels off for $r<h$, so that the potential will have little structure at scales less than the vertical scale height $h$.

How well can one estimate the potential? The following things can affect the estimate: the vertical scale height, the choice of vertical density function, the wavelength of image used to trace mass, the bulge-disk decomposition, and projection effects caused by the viewing angle of the galaxy (not discussed here). In this section we discuss the decomposition of the potential into its Fourier components or moments so that each moment can be described in terms of an angle and a magnitude.

Vertical Scale Height and Choice of Vertical Density Function Studies of edgeon disks in the IR find for a galaxy light density $\propto e^{-R / h_{d}} e^{-z / h}$ that the ratio of scale lengths $q_{0} \equiv h / h_{d} \sim 1 / 12$. We used values for the vertical scale height determined from this relation as a starting point for our estimate of the potential. We note that it is likely that vertical scale lengths are correlated with Hubble type and that galaxies of an individual Hubble type should have a range of $q_{0}$ (Fouqué et al 1990). We note that values for $q_{0}$ given by Fouqué et al. (1990) were much larger than those found in optical studies of edge-on disks which were larger than those found from IR studies of edge-on disks (see references in Quillen et al. 1994). Hopefully modeling of the dust and multi-band statistical surveys will be able to resolve these uncertainties.

We estimated the gravitational potential in a few barred galaxies to determine the effect of varying the vertical scale height. We found that doubling the scale height typically affected the strength of the moments at the $10-15 \%$ level and had little effect upon the angles of the moments. We also found that the potential is insensitive (moments are affected less than $1-2 \%$ ) to the choice of vertical density function for functions with the same $<z^{2}>$ (Quillen \& Athanassoula 1995).

Wavelength of Image Because of young stars and absorption from dust we found a large difference in the strength of the moments as well as the angles between potentials generated from optical images and potentials generated from infrared images. Late-type galaxies usually have optical to infrared radial color gradients in the sense that they become bluer with increasing radius (deJong 1995). This means that IR generated maximal disk rotation curves drop faster than optically generated ones implying that more dark matter is needed at smaller radii than previously found from optical studies. I and Heraudeau et al. (1995) find from a dozen or so galaxies that $K$ band mass-to-light ratios range from $1.0-1.4 M_{\odot} / L_{K \odot}$.

Dwarfs can have color gradients in the sense that the galaxy becomes redder with increasing radius (e.g. in NGC 1705, Quillen et al. 1995b) so that dwarf mass models may not necessarily need to be as halo dominated as previously found. Dwarfs also typically have significantly lower $V-K$ colors than larger galaxies so that infrared mass-to-light ratios for dwarf maximal disk models may be low, much closer to those found for larger late-type galaxies. These results are somewhat premature; clearly more careful dynamical and multi-band studies of dwarfs need to be done. 

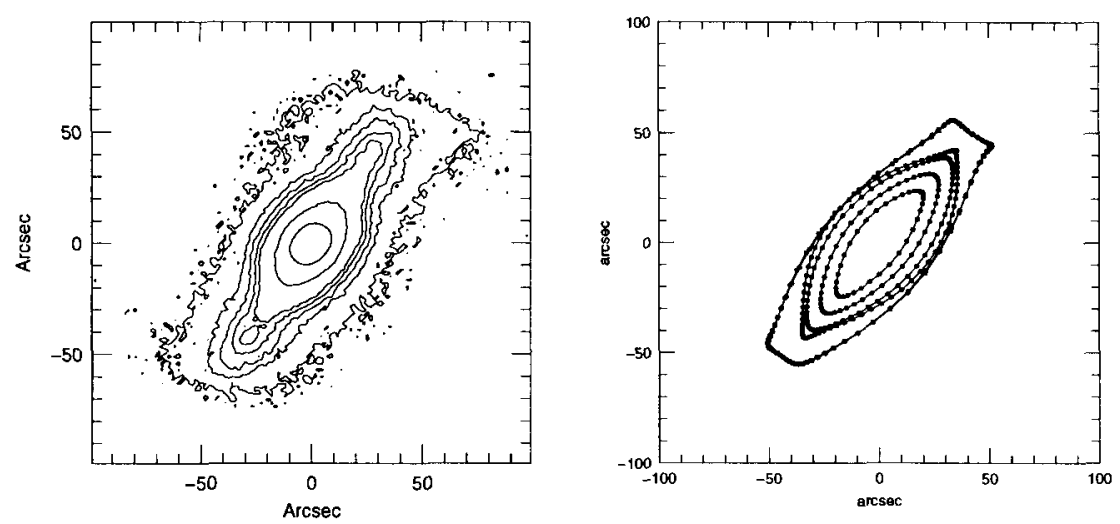

Figure 3. The left hand side shows contours of the $K(2.2 \mu \mathrm{m})$ image of the face-on barred galaxy NGC 4314. The right hand side shows prograde closed orbits in the frame in which the bar is stationary. These orbits are predicted from the gravitational potential which was estimated from the infrared image. Points are plotted at equal time intervals in a single orbit. The density of the galaxy should increase where the velocity decreases.

\section{Orbits in NGC 4314 and NGC 7479}

NGC 4314 is a nice galaxy in which to integrate orbits because it has a small bulge (making it unnecessary to do a bulge-disk decomposition), and it is close to face-on so that no correction for the inclination is necessary. The near-IR colors are constant almost everywhere, so we do not need to correct for changes in the mass-to-light ratio. NGC 4314's high symmetry about the origin, lack of gas, and weak spiral arms imply that the bar is close to stable. NGC 4314 also has prominent peaks in intensity near the end of its bar (which we refer to as knobby ends to the bar) which we would like to understand (see Figure 3).

Two parameters affect the shape of closed orbits inside corotation: $h$, the vertical scale height and $\Omega_{b}$ the bar pattern speed. We found that if we placed the corotation radius near the end of the bar, that there was an $m=4$ Inner Lindblad resonance near the location of the knobby features near the end of the bar. The smaller the scale height, the more structure is present in the potential so that $h$ affects ellipticity of orbits almost everywhere, whereas changing $\Omega_{b}$ moves the location of the resonances and causes large changes in the shapes of the orbits only near the resonances. Because of this we found that we could constrain both parameters.

We found that closed orbits within the $m=4$ resonance were lozengeshaped and had pronounced peaks near the end of the bar with low speeds (implying high density) nearest the $m=4$ resonance. This implies that the knobby features at the ends of the bar are caused by the shape of these most peaked lozenge-shaped orbits (see Figure 3 ). We also found that the spiral arms began very close to the location of the $m=4$ resonance. Studies of other galaxies should determine if this is true in general. 
It would be interesting to consider the ellipticity of orbits as a function of radius in late type bars as compared to early type bars. I suspect that the reason early type bars have shoulders in their profile along the major axis is because the orbits have large changes in ellipticity as a function of radius. Late type bars should have a large separation between resonances, and orbits with constant ellipticity as a function of radius so that they have exponential profiles along their major axis.

I also tried integrating closed orbits in NGC 7479 which is highly asymmetric. It was much more difficult to find the closed orbits because of the lack of symmetry in this galaxy. The $\mathrm{m}=1,3,5$ moments increase the number of resonances and also cause bifurcations in the closed orbit families (Patsis 1995). I found it difficult to find orbits that looked like the galaxy for a range of pattern speeds and vertical scale heights. Possible explanations are that I had bad estimates for the potential, or that the system is evolving quickly. Alternatively ergodic orbits may conspire to support the pattern so that there are few closed orbits that resemble the galaxy.

\section{Torque on the Gas and the Gas Inflow Rate Along the Bar in NGC 7479}

NGC 7479 is a strongly barred galaxy with star formation occurring along its bar. Its highly asymmetrical spiral arms suggest that it is in a transient or quickly evolving state. In fact, the appearance of the galaxy is similar to the galaxy displayed in the minor merger simulation of Mihos \& Hernquist (1994) during the time when gas inflow occurs. In NGC 7479 the molecular gas (traced in $\mathrm{CO}$ emission) is coincident with emission in $\mathrm{H} \alpha$ and lies in a linear feature, running along, but slightly offset from the center of the bar as seen in the near-IR images (see Figure 4). This implies that there is a torque on the gas. Hydrosimulations of gas flow in barred galaxies find that the gas in these linear features is stationary in the frame in which the bar is still, so that the angular momentum in the gas can be approximated as $l \sim r^{2} \Omega_{b}$ and the torque $d l / d t \sim 2 r \Omega_{b} d r / d t$. The gas inflow rate can be estimated as $\frac{d r}{d t}=\frac{d \Phi / d \theta}{2 r \Omega_{b}}$, so that an estimate of the azimuthal derivative of the gravitational potential at the location of the gas emission can be used to estimate the gas inflow rate. We note that inflow is difficult to determine from velocities observed in the gas because they show large streaming motions. Using our estimate for the gravitational potential we derive a gas inflow rate in NGC 7479 of $10-20 \mathrm{~km} \mathrm{~s}^{-1}$ which corresponds to a mass inflow rate of $4 M_{\odot}$ per year. We note that this estimate is roughly consistent with that observed in Mihos \& Hernquist's (1994) SPH simulation but is somewhat higher than that observed in Athanassoula's hydro simulations.

We eagerly await results of simulations (listed above) to see what values they find are consistent with the observations. In particular we hope that these simulations can constrain what kind of ISM might be consistent with the observations. High inflow rates are not inconsistent with SPH simulations but are large compared to those predicted by sticky particle and hydro simulations. We suggest that the low dissipation required for ring formation is not necessarily inconsistent with high inflow rates along bars if the form of dissipation required 

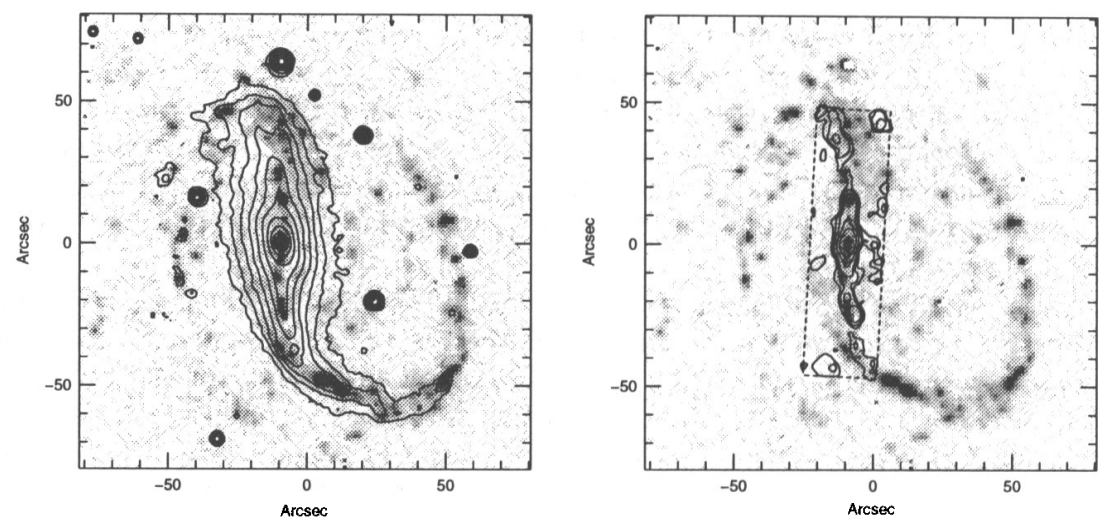

Figure 4. The left hand side shows an $\mathrm{H} \alpha$ emission gray scale and $K$ $(2.2 \mu \mathrm{m})$ contour overlay of the asymmetric barred galaxy NGC 7479 . Note that the $\mathrm{H} \alpha$ is offset from the center of the bar. The right hand side shows an $\mathrm{H} \alpha$ emission gray scale and $\mathrm{CO}(1-0)$ emission contour overlay. Note that the $H \alpha$ and $\mathrm{CO}$ are almost coincident. The offset of these emission features from the center of the bar imply that there is a torque on the gas. This torque, estimated from the gravitational potential (derived from the infrared image) implies a gas inflow rate along the bar of $10-20 \mathrm{~km} \mathrm{~s}^{-1}$.

to simulate the flow is dependent upon the shear and compression observed in these shocks.

Acknowledgments. This work was done in collaboration with the OSU galaxy survey group (R. W. Pogge, D. L. DePoy, J. A. Frogel, R. A. González, S. V. Ramírez, D. M. Terndrup, L. E. Kuchinski, G. Tiede, K. Sellgren, R. Davies), and also J. D. Kenney, and E. Athanassoula. I am grateful to the Observatoire de Marseille for its hospitality and support during Spring 1995.

\section{References}

Athanassoula, E. 1991, in Dynamics of Disk Galaxies, B. Sundelius, Göteburg: Chalmers University, 149

De Jong, R. S. 1995, Ph.D. Thesis, Rijksuniversiteit Groningen

Fouqué, P., Bottinelli, L., Gouguenheim, L., \& Paturel, G. 1990, ApJ, 349, 1

Frogel, J. A. 1988, ARA\&A, 26, 51

Gnedin, O., Goodman, J. \& Rhoads, J. 1995, ESO/MPA Workshop on Spiral Galaxies in the near IR, ESO Garching b. Munchen

Heraudeau, Ph., Simien, F., \& Mamon G. 1995, ESO/MPA Workshop on Spiral Galaxies in the near IR, ESO Garching b. Munchen

Kent, S. M. \& Glaudell, G. 1989, AJ, 98, 1588

Mihos, J. C. \& Hernquist, L. 1994, ApJ, 425, L13 
Quillen, A. C., Frogel, J. A., \& González, R. A. 1994, ApJ, 437, 162

Quillen, A. C., Frogel, J. A., Kenney, J. D, Pogge, R. W., \& DePoy, D. L. 1995a, ApJ, 441, 549

Quillen, A. C., Ramírez, S. V., \& Frogel, J. A. 1995b, ApJ, 110, 205

Quillen, A. C. \& Athanassoula, E. 1995, in preparation

Patsis, P. 1995, ESO/MPA Workshop on Spiral Galaxies in the near IR, ESO Garching b. Munchen.

\section{Discussion}

E. Athanassoula: Your inflow rate is an upper limit because the whole fluid flow has not been considered.

P. Teuben: The velocity dispersion will influence the ellipticity of the isophotes.

D. Pfenniger: I did estimate the scale height as a function of radius in $\mathrm{N}$-body bar models. The result is counter-intuitive: the scale height decreases by about a factor of 2 in the bulge with respect to the stellar disk. 